\title{
Modeling of changes in heat resistance of nickel-based alloys using Bayesian artificial neural networks
}

\author{
O. V. Anoshina ${ }^{\dagger, 1}$, A. S. Trubnikova 2 , O. B. Milder ${ }^{2}$, D. A. Tarasov², \\ A. A. Ganeev ${ }^{3}$, A. G. Tyagunov ${ }^{2}$ \\ †anoshina@inbox.ru

\begin{abstract}
${ }^{1}$ Russian State Vocational Pedagogical University, 11 Mashinostroiteley St., Yekaterinburg, 620012, Russia ${ }^{2}$ Ural Federal University n. a. the first President of Russia B. N. Yeltsin, 19 Mira St., Yekaterinburg, 620002, Russia ${ }^{3}$ Ufa State Aviation Technical University, 12 K. Marx St., Ufa, 450008, Russia
\end{abstract}

Resource design of gas turbine engines and installations requires extensive information about the heat resistance of nickel-based superalloys, from which the most critical parts of aircraft and marine engines, pumps of gas-oil pumping stations and power plants are made. The problems are that the data on the heat resistance obtained as a result of testing each alloy under study are quite limited. In the present paper, the task of modelling changes in the heat resistance of nickel-based superalloy on the basis of available experimental data is solved. To solve the task, the most modern approach, the neural network modeling method, was applied. The input data are chemical compositions of heat-resistant nickel-based superalloys and the values of their heat resistance obtained experimentally. The output data are the calculated values of heat resistance modeled by an artificial neural network. In the course of the work, transformations of the input data were carried out to reduce the standard deviation of the modeling of the output data. The choice of the neural network configuration was made in order to achieve the highest possible accuracy. As a result, a neural network of direct error propagation was used, with 27 neurons on the input layer, 13 neurons in the hidden layer and 1 neuron in the output layer. To validate the results of the predictions, a group of alloys with the maximum number of known experimental values of heat resistance was randomly selected before the input of data into the network. After preparing the data, selecting the configuration and training the network, the chemical compositions of the selected group were loaded and their heat resistance values were calculated. Comparison of the obtained data with the experimental data showed high efficiency of the method. As a result, data on the change of heat resistance for the studied alloys were obtained and an analytical expression describing the obtained dependences was formulated.

Keywords: neural networks, nickel-based superalloys, heat resistance, thermal stability.

УДК: 620.22

\section{Моделирование изменения предела длительной прочности сплавов на основе никеля с использованием байесовских искусственных нейронных сетей}

\author{
Аношина О.В. ${ }^{\dagger, 1}$, Трубникова А.С. ${ }^{2}$, Мильдер О.Б. ${ }^{2}$, Тарасов Д. А. ${ }^{2}$, \\ Ганеев А. А. ${ }^{3}$, Тягунов А. Г. ${ }^{2}$ \\ ${ }^{1}$ Российский государственный профессионально-педагогический университет, \\ ул. Машиностроителей, 11, Екатеринбург, 620012, Россия \\ ${ }^{2}$ Уральский федеральный университет им. первого президента России Б. Н. Ельцина, \\ ул. Мира, 19, Екатеринбург, 620002, Россия \\ ${ }^{3}$ Уфимский государственный авиационный технический университет, ул. К. Маркса, 12, Уфа, 450008, Россия
}

Ресурсное проектирование газотурбинных двигателей и установок требует расширенных сведений о жаропрочности никелевых сплавов, из которых изготавливаются наиболее ответственные детали авиационных и судовых двигателей, насосов газонефтеперекачивающих станций и энергетических установок. Проблемы заключаются в том, что данных о жаропрочности, полученных в результате испытаний каждого исследуемого сплава, достаточно 
мало. Задачи, решаемые в настоящей работе, следующие: смоделировать изменения жаропрочности никелевых сплавов на основе имеющихся экспериментальных данных. Для решения поставленных задач применен наиболее современный подход - метод нейросетевого моделирования. Входными данными являются химические составы жаропрочных никелевых сплавов и значения их жаропрочности, полученные экспериментально. Выходными данными стали расчётные значения жаропрочности, смоделированные искусственной нейронной сетью. В ходе работы проведены преобразования входных данных для снижения среднеквадратичного отклонения моделирования выходных данных. Выбор конфигурации нейронной сети производился с целью достижения максимально возможной точности. В итоге использована нейронная сеть прямого распространения ошибки, с 27 нейронами на входном слое, 13 нейронами в скрытом слое и 1 нейроном на выходном слое. Для валидации результатов предсказаний еще до ввода данных в сеть случайным образом была отобрана группа сплавов с максимальным количеством известных экспериментальных значений жаропрочности. После подготовки данных, выбора конфигурации и обучения сети были загружены химические составы выделенной группы и рассчитаны значения их жаропрочности. Сравнение полученных данных с экспериментальными показали высокую эффективность метода. В результате получены данные об изменении жаропрочности для исследованных сплавов, а также сформулировано аналитическое выражение, описывающее полученные зависимости.

Ключевые слова: нейронные сети, никелевые сплавы, жаропрочность, термостабильность.

\section{1. Введение}

Изделия из жаропрочных никелевых сплавов (ЖНС) нашли широкое применение в газотурбинных двигателях, насосах газо-нефтеперекачивающих станций и энергетических установках. Комплекс эксплуатационных свойств обеспечивается оптимальной микроструктурой сплавов. Поскольку работа в условиях высоких температур сопровождается большими нагрузками, в изделиях происходит деградация структуры и, следовательно, трещинообразование и излом. В этой связи к жаропрочным сплавам предъявляются особые требования, среди которых наиболее важными являются жаропрочность [1-3].

Жаропрочность - это способность материала длительное время сопротивляться (не поддаваться деформации или разрушению) нагрузкам при высоких температурах [4]. Жаропрочность никелевых сплавов оценивается пределами ползучести или длительной прочности при высоких температурах [1]. В данной работе использованы результаты испытаний на предел длительной прочности [5]. Температурновременные режимы выдержек задаются при ресурсном проектировании конкретного изделия и не охватывают все возможные диапазоны. Данный факт составляет одну из проблем, поскольку сложно оценить возможность применения сплавов для изделий, эксплуатирующихся при других температурах.

Таким образом, в настоящей работе поставлена следующая задача: предложить и апробировать метод моделирования изменения предела длительной прочности жаропрочных никелевых сплавов на основе экспериментальных данных.

Для сравнения результатов испытаний образцов на предел длительной прочности, используется величина, отражающая одновременно температуру и продолжительность нахождения при нагрузке параметр Ларсона - Миллера (1) [6]

$$
P_{L M}=T(20+\lg \tau) / 1000,
$$

где $T$ - температура (К), $\tau-$ продолжительность нахождения при нагрузке (ч).

\section{2. Методика}

Для решения, поставленных в настоящей работе, задач возможно использование метода искусственных нейронных сетей (ИНС), The MATLAB Neural Network Toolbox $[7,8]$, как современного подхода, учитывающего результаты многочисленных испытаний образцов сплавов с различными химическими составами. ИНС моделирует связь между входными и выходными данными, используя определенные нелинейные зависимости [8]. Так как ИНС является нелинейным статистическим методом, он может быть использован для решения трудно формализуемых задач [9].

В выбранной модели ИНС прямого распространения (Рис. 1) сигналы передаются в одном направлении от входного слоя к выходному. Обучение искусственной нейронной сети состоит в том, чтобы посредством подачи на входы сети обучающего воздействия и, таким образом, изменения весов и преобразования сигналов внутри сети определить закономерность между входными и выходными данными, выражающуюся в функциональной зависимости.

Входные данные были взяты из производственной базы данных, объединяющей наиболее полную

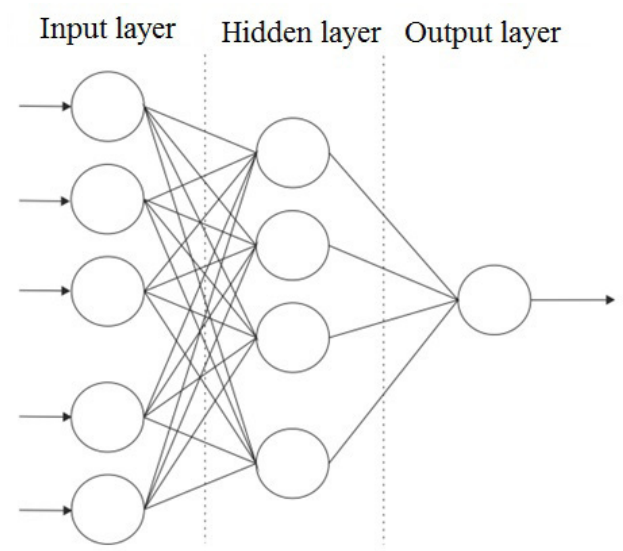

Рис. 1. Модель искусственной нейронной сети прямого распространения.

Fig. 1. Model of artificial neural network of direct propagation. 
экспериментальную информацию о жаропрочных никелевых сплавах. Для вычислительного эксперимента была выбрано 210 марок только монокристаллических жаропрочных никелевых сплавов, как отечественных, так и зарубежных. Химические составы и свойства 200 марок ЖНС были использованы для обучения сети и получения модели, а оставшиеся десять составов со свойствами использовались для оценки точности (валидации) полученной модели.

Для обработки данных был использован многослойный перцепетрон с прямой связью. Так как поставленная задача является нелинейной, а функция непрерывно убывающей, выбор данной модели ИНС является оптимальным [10].

Перед началом обучения ИНС, необходимо было провести подготовку данных [8], которая заключалась в исключении из обучения марки сплавов с неполными данными (отсутствие более $20 \%$ параметров) и в нормировании параметров относительно своего максимума, так как входные данные существенно различались как по единицам измерения, так и по масштабу шкал.

Так как предел длительной прочности не может быть отрицательным числом, а разность минимального и максимального значений этого параметра достигает нескольких порядков, было принято решение преобразовать эти данные по формуле [11]:

$$
y_{\sigma}=-\lg \left(\sigma_{T}^{\tau}\right)
$$

где $\sigma$ - предел длительной прочности (МПа).

Такое преобразование имеет ряд преимуществ. Во-первых, диапазон пределов длительной прочности покрывает несколько порядков величины (от 20-25 до $1000 \mathrm{MПа)} \mathrm{и} \mathrm{на} \mathrm{графиках} \mathrm{зависимости}$ от параметра Ларсона-Миллера традиционно изображается в логарифмическом масштабе. Во-вторых, использование логарифма делает ошибку прогноза относительной во всем диапазоне $\sigma$. В-третьих, обратное преобразование

$$
\sigma_{T}^{\tau}=10^{-y_{\sigma}}
$$

исключает возможность получения отрицательных значений прогнозируемой величины на нижней границе диапазона пределов длительной прочности.

Знак «минус» в формуле (3) имеет целью встроить в процесс обучения сети априорную информацию: поскольку цель - нахождение зависимости предела длительной прочности от параметра Ларсона-Миллера, каковая является убывающей, то и преобразование выходной величины описывается убывающей функцией.

После всех преобразований, в качестве входных данных использовали концентрации 25 химических элементов (C, Cr, Co, Mo, W, Al, Ti, Nb, B, Fe, Y, Zr, Ta, $\mathrm{Re}, \mathrm{Ru}, \mathrm{V}, \mathrm{Ce}, \mathrm{La}, \mathrm{S}, \mathrm{Si}, \mathrm{Mn}, \mathrm{Mg}, \mathrm{P}, \mathrm{Hf}, \mathrm{Si})$ в 210 марках монокристаллических жаропрочных никелевых сплавах и условия испытаний на длительную прочность, характеризующиеся температурой испытаний (К) и продолжительностью нахождения под нагрузкой (ч). В качестве выходных данных - предел длительной прочности для каждого состава сплава. Таким образом, задачей ИНС являлось определение корреляции между химическим составом жаропрочных никелевых сплавов, условиями испытаний и пределом длительной прочности.

Метод машинного обучения заключается в построении искусственной нейронной сети в прикладом пакете Matlab; поиск закономерностей соответствия химического состава жаропрочных никелевых сплавов пределу длительной прочности, полученному в результате испытаний; оценка полученных корреляций c использованием тестовой выборки; определение точности модели по относительной среднеквадратичной ошибке (RMSE). Чем меньше среднеквадратичное отклонение, тем точнее результат [11]

$$
R M S E=\sqrt{\frac{\sum_{i=1}^{n}\left(y_{\text {modeli }}-y_{i}\right)^{2}}{n}},
$$

где $y_{\text {modeli }}-$ смоделированные значения предела длительной прочности, $y_{i}-$ экспериментальные значения предела длительной прочности, $n-$ количество данных предела длительной прочности.

На этапе создания ИНС, после проведения ряда проб, было решено использовать ИНС прямого распространения ошибки, с 27 нейронами на входном слое, 13 нейронами в скрытом слое и 1 нейроном на выходном слое (Рис. 2). Число нейронов в скрытом слое подбиралось эмпирически по критерию уменьшения RMSE на полной выборке из 200 марок ЖНС. Исходно, число нейронов в скрытом слое было выбрано равным пяти и в каждом последующем вычислительном эксперименте увеличивалось на один. В рамках каждого вычислительного эксперимента одновременно создавалось пять идентичных сетей, которые независимо друг от друга тренировались на полной выборке. В окончательном варианте сети в скрытом слое было 13 нейронов.

Для борьбы с одной из основных проблем в обучении нейронных сетей - переобучением - был применен специализированный «бутстрэп»-алгоритм. Процесс тренировки сети был разбит на итерации (до 500). Каждая итерация состоит из небольшого (до 30) количества эпох (в терминологии ИНС). На каждой итерации из общей выборки случайным образом

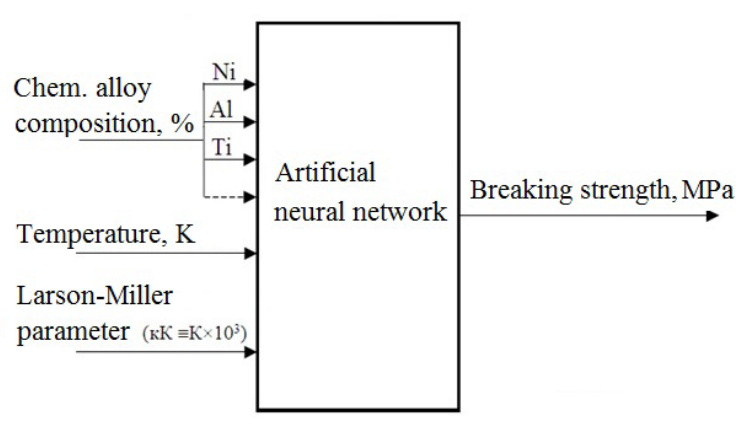

Рис. 2. Схематическая модель ИНС для моделирования изменения предела длительной прочности сплавов на основе никеля.

Fig. 2. Schematic model of artificial neural network for modeling changes in the limit of long-term strength of nickel-based alloys. 
извлекается без повторений 90\% образцов, которые и используются для обучения сети на этапе текущей итерации. Эти образцы разбиваются на собственно тренировочную (75\%) и внутреннюю тестовую группы (25\%). Критерием выхода из процесса обучения на этапе каждой итерации является достижение целевого значения RMSE. Целевое значение RMSE подбирается эмпирически и устанавливается чуть ниже уровня, при котором начинают расходиться RMSE тренировочной и тестовой выборки, полученные после очередной эпохи обучения. По завершении процесса обучения на данной итерации образцы возвращаются в общую выборку.

Таким образом, сеть многократно «доучивается» на варьируемом наборе до тех пор, пока значение RMSE, полученное на тестовой выборке, превышает значение RMSE, полученное на тренировочной выборке на величину более, чем 20\%. В итоге среднеквадратичная ошибка была получена на уровне 15\%. Далее модель ИНС использовалась для получения зависимостей предела длительной прочности от параметра Ларсона-Миллера для сплавов валидационной группы (Рис. 3).

\section{3. Результаты и их обсуждение}

Графики зависимостей $\sigma=f\left(P_{L M}\right), \quad$ представленные на Рис. 3 свидетельствуют - адекватность результатов обеспечивается удовлетворительной сходимостью предсказанных и реальных значений. Обобщенный вид зависимости представлен на Рис 4. Предел длительной прочности уменьшается с увеличением параметра Ларсона-Миллера, ход кривой имеет одинаковый
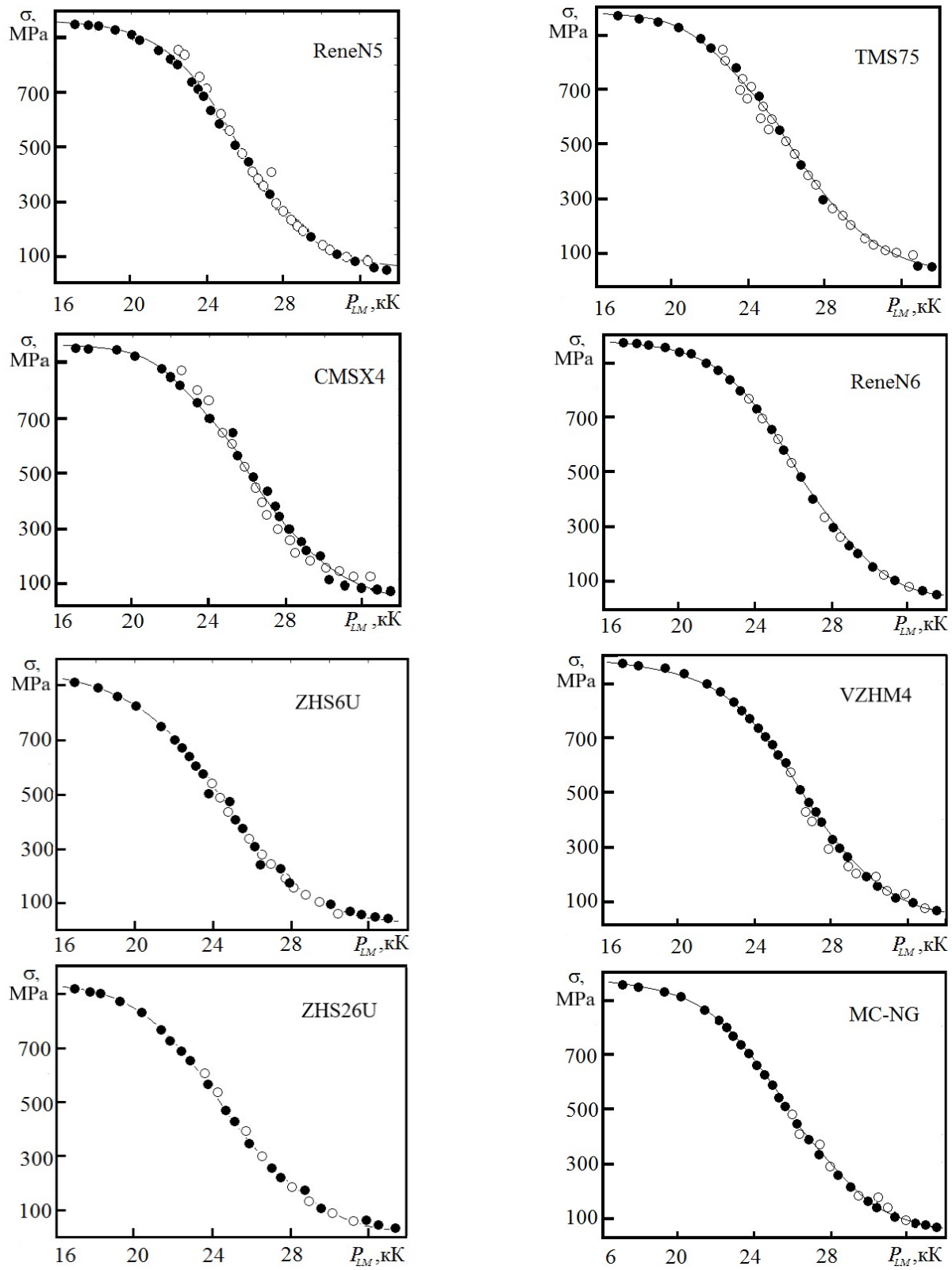

Рис. 3. Зависимости пределов длительной прочности сплавов на основе никеля от параметра Ларсона-Миллера: о - экспериментальные значения; - - спрогнозированные значения.

Fig. 3. Dependences of the limits of long-term strength of nickel-based superalloys on the Larson-Miller parameter: o - experimental values; $\bullet$ - predicted values. 


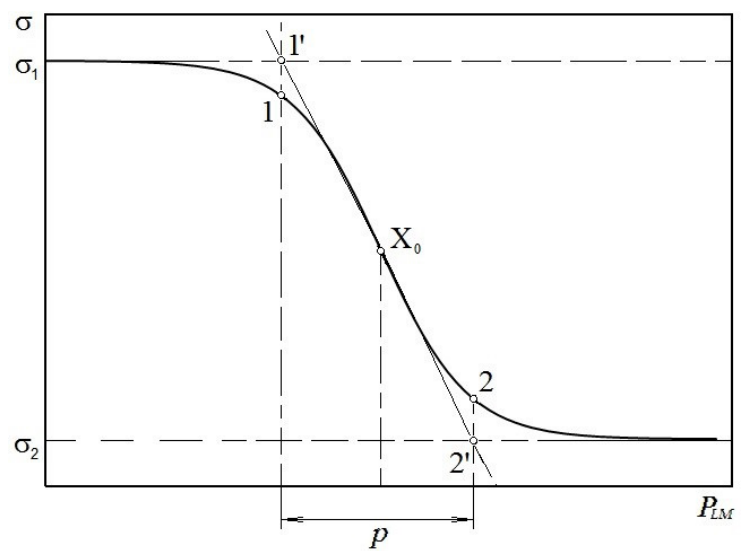

Рис. 4. Обобщенный вид зависимости $\sigma=f\left(P_{L M}\right)$ жаропрочных никелевых сплавов.

Fig. 4. The generalized dependence of the $\sigma=f\left(P_{L M}\right)$ of nickel-based superalloys.

s-образный вид, характерный для всех изученных сплавов. Кривая зависимости не монотонна - имеет характерные для каждого состава сплава особенности: точки перегибов, обозначенные как 1 и 2 и участок с наиболее интенсивным убыванием предела длительной прочности. Характерные точки перегибов и угол наклона участка с интенсивными убыванием $\sigma$ зависят от структурных изменений, происходящих в сплавах в период испытаний. Наши исследования $[12,13]$ и результаты других ученых [14-21] позволяют представить общий порядок этих структурных изменений: после литья и термической обработки структура монокристаллических ЖНС состоит из $\gamma$-никелевой матрицы, основной упрочняющей мелкодисперсной $\gamma^{\prime}$-вторичной фазы и эвтектики $\left(\gamma+\gamma^{\prime}\right)$. Температура и приложенные во время испытаний нагрузки приводят к существенным структурным изменениям: образуется рафт-струкутра и возникают дислокации; кубоидные выделения основной упрочняющей фазы коагулируют, происходит рост их блоков; при выдержках свыше $1100^{\circ} \mathrm{C}$ количество $\gamma^{\prime}$-вторичной фазы существенно снижается; округлые выделения $\gamma+\gamma^{\prime}$ эвтектики заменяются на колонии мелких частиц; структурные изменения приводят к возникновению и росту охрупчивающих топологически плотно упакованных фаз, типа $\mu, \sigma$, Лавеса и других. Все это приводит к разупрочнению структуры и хрупкому излому.

Зависимость $\sigma=f\left(P_{L M}\right)$ представляет собой две соединенные экспоненты и описывается аналитической функцией, для упрощения примем $x \equiv P_{L M}$,

$$
\sigma(x)=\sigma_{2}+\frac{\sigma_{1}-\sigma_{2}}{1+\exp \left(\frac{x-x_{0}}{p}\right)},
$$

где, $\sigma_{1}, \sigma_{2}, x_{0}$ и $p$ параметры, устанавливаемые в ходе аппроксимации зависимости $\sigma=f\left(P_{L M}\right)$ для конкретного состава сплава. $\sigma_{1}-$ максимальное значение предела длительной прочности, $\sigma_{2}-$ минимальное значение предела длительной прочности, $x_{0}-$ значение параметра Ларсона-Миллера в середине участка интенсивного уменьшения предела длительной прочности; $p$ - коэффициент наклона касательной в точке $\mathrm{X}_{0}$.

\section{4. Выводы}

Предложен метод моделирования изменения предела длительной прочности жаропрочных никелевых сплавов от температурно-временных условий испытаний, заключающийся в установлении корреляций между химическими составами и значениями предела длительной прочности применением искусственных нейронных сетей. Погрешность метода не превышает $15 \%$.

Апробация модели на жаропрочных никелевых сплавах, не принимавших участие в получении модели подчеркнула точность предложенного метода.

Зависимости $\sigma=f\left(P_{L M}\right)$ имеют одинаковый немонотонный характер понижения предела длительной прочности от температурно-временных условий испытаний, но для каждого состава сплава имеются характерные особенности: координаты точек перегиба кривых и угол наклона участка с наиболее интенсивным убыванием значений предела длительной прочности.

Для описания зависимостей $\sigma=f\left(P_{L M}\right)$ жаропрочных никелевых сплавов предложена аналитическая функция (5).

\section{Литература/References}

1. Ch. T. Sims. Superalloys II: High-Temperature Materials for Aerospace and Industrial Power (ed. by Ch. T. Sims, N. S. Stoloff, W. K. Hagel). New York, Wiley (1987) 640 p.

2. C.R. Reed. The Superalloys. Fundamentals and Applicatios. Cambridge University Press (2006) 372 p. Crossref https://doi.org/10.1017/CBO9780511541285

3. E.N. Kablov, V.V. Gerasimov, E.M. Visik. Aviation Industry. 2, 18 (1999). (in Russian) [Е.Н. Каблов, В. В. Герасимов, Е. М. Висик. 2, 18 (1999).]

4. Yu. M. Lakhtin, V.P. Leontyev. Materialovedeniye. Moscow, Mashinostroenie (1990) pp. 16-18. (in Russian) [Ю.М. Лахтин, В.П. Леонтьева. Материаловедение Москва, Машиностроение (1990) с. 16-18.]

5. GOST 10145-81 Metals. Metod ispytaniya na dlitel'nuyu prochnost'. Moscow, Izdatel'stvo standartov (1981). (in Russian) [ГОСТ 10145-81 Металлы. Метод испытания на длительную прочность. Москва, Издательство стандартов (1981).]

6. B. N. Sinayski, M. S. Belyaev, N. D. Zhukov, A. D. Panteleev. Strength of Materials. 21, 322 (1989). Crossref

7. M.H. Beale, M. T. Hagan, H. B. Demuth. Matlab Neural Network Toolbox User's Guide. Mathworks (2018) 558 p.

8. S. Khaikin. Neyronnyye seti: Polnyy kurs. Moscow: Williams (2017) 1104 p. (in Russian) [C. Хайкин. Нейронные сети: Полный курс. Москва, Вильямс (2017) 1104c.]

9. P.D. Wasserman. Neural Computing: Theory and Practice. Coriolis Group (1989) 230 p.

10. R. Callan. Osnovnyye kontseptsii neyronnykh setey. Moscow, Williams (2001) 288 p. (in Russian) [Р. Каллан. Основные концепции нейронных сетей. Москва, Вильямс (2001) 288 с.]

11. G.I. Ivchenko, Yu.I. Medvedev. Matematicheskaya statistika. Textbook. Moscow, Liberkom (2014) 352 p. (in Russian) [Г.И. Ивченко, Ю.И. Медведев. 
Математическая статистика. Учебник. Москва, Либерком (2014) 288 с.]

12. A. G. Tyagunov, E. E. Baryshev, T. K. Kostina, B. A. Baum, V.P. Lesnikov, I. P. Semenova. Phys. Metal. Metallogr. 86, 65 (1998).

13. A.G. Tyagunov, E.E. Baryshev, T.K. Kostina, I.P. Semenova, V.P. Lesnikov. Metal Science and Heat Treatment. 41, 538 (1999). $\underline{\text { Crossref }}$

14. V.P. Kuznetsov, V.P. Lesnikov, N.A. Popov. Struktura i svoystva zharoprochnykh nikelevykh splavov. Publishing house of the Ural University (2016) 164 p. (in Russian) [В.П. Кузнецов, В.П. Лесников, Н.А. Попов. Структура и свойства жаропрочных никелевых сплавов. Издательство Уральского университета (2016) 164 c.]

15. E. N. Kablov. Lityye lopatki gazoturbinnykh dvigateley. Splavy, tekhnologiya, pokrytiya. Moscow, MISIS (2001) 632 p. (in Russian) [Е.Н. Каблов. Литые лопатки газотурбинных двигателей. Сплавы, технология, покрытия. Москва, МИСИС (2001) 632 с.]

16. A.V. Logunov, Yu.N. Smolin. Sovremennyye zharoprochnyye nikelevyye splavy dlya diskov gazovykh turbin. Moscow, Nauka I Tekhnologii (2013) 264 p. (in Russian) [А.В. Логунов, Ю.Н. Шмотин. Современные жаропрочные никелевые сплавы для дисков газовых турбин. Москва, Наука и технологии (2013) 264 с.]

17. S. V. Gaiduk, T. V. Tikhomirova. Aerospace engineering and technology. 9, 33 (2015). (in Russian) [C. В. Гайдук, Т.В. Тихомирова. Авиационно-космическая техника и технология. 9, 33 (2015).]

18. S. V. Gaiduk, V.V. Kononov, V. V. Kurenkova. General issues of metallurgy. 4, 31 (2015). (in Russian) [С. В. Гайдук, В.В. Кононов, В.В. Куренкова. Общие вопросы металлургии. 4, 31 (2015).]

19. S. V. Gaiduk, V.V. Kononov, V.V. Kurenkova. Electrometallurgy today. 4 (129), 44 (2017). (in Russian) [C. В. Гайдук, В. В. Кононов, В.В. Куренкова. Современная электрометаллургия. 4 (129), 44 (2017).] Crossref

20. E. N. Kablov, O.G. Ospennikova, N.V. Petrushin, E. M. Visik. Aviation materials and technologies. 2, 14 (2015). (in Russian) [Е.Н. Каблов, О.Г. Оспенникова, Н.В. Петрушин, Е.М. Висик. Авиационные материалы и технологии. 2, 14 (2015).] Crossref

21. E. N. Kablov, O. G. Ospennikova, N. V. Petrushin. Aviation materials and technologies. 1, 34 (2015). (in Russian) [Е.Н. Каблов, О.Г. Оспенникова, Н.В. Петрушин. Авиационные материалы и технологии. 1, 34 (2015).] $\underline{\text { Crossref }}$ 